\title{
MS02-P02 | GETTING THE MOST FROM YOUR CRYSTALS THROUGH THE FULL AUTOMATION OF
}

COMPLEX EXPERIMENTS

Bowler, Matthew (European Molecular Biology Laboratory, Grenoble, FRA)

has been a key development in macromolecular crystallography allowing the remote operation of beamlines and the ability to screen thousands of samples and process the subsequent deluge of data. The full automation of data collection, where the process of mounting, locating, characterising and collecting data is left entirely to algorithms, is possible on the ESRF beamline MASSIF-1 [1,2]. In contrast to existing automatic beamlines, where automation is confined to mounting and loop centring of samples, MASSIF-1 uses mesh scans and characterisation combining data gathered during the process, such as crystal volume, with user input to calculate optimised strategies. The ability to make complex decisions at multiple stages of the location and characterisation phases mean that any sample can be processed in a wide variety of mounts allowing even the most challenging samples to be treated automatically.

The full automation of data collection from crystals of biological macromolecules opens the door to large scale screening for all laboratories without significant person hour investments. Projects from screening large numbers of highly variable crystals to small molecule fragment campaigns are now possible with thousands of data sets collected in a consistent manner day and night, often with better quality than human operators [3]. In combination with developments in the robotic mounting and soaking of crystals [4] we envision that the future of macromolecular crystallography is the provision of a fully automated high throughput service able to rapidly produce high quality structural models and screen for potential therapeutic and probe molecules. 